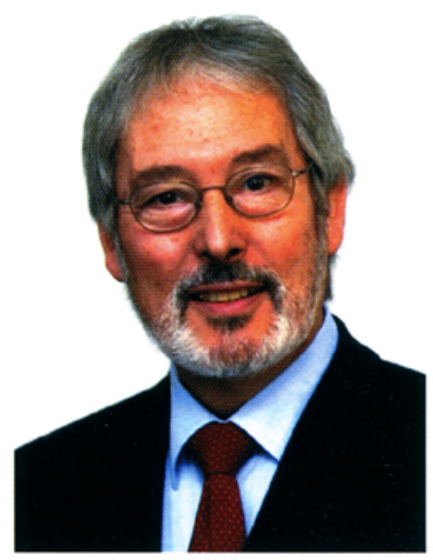

Rolf Harbeck

\section{Von Information und Kommunikation}

Liebe Leserin, lieber Leser,

empfinden Sie es auch so? In immer kürzeren Abständen werden wir mit spektakulären Neuerungen konfrontiert, die sich auf das tägliche Leben auswirken. Wir nehmen wahr, wie sich eine neve Arbeits- und Alltagskultur entwickelt, in der das computergesteuerte System die manuelle Produktionskraft des Menschen verdrängt und ihm intellektuelles Agieren abnimmt, ihn aber gleichzeitig zu neuen Denk- und Entwicklungsleistungen herausfordert. Wir nutzen Informations- und Kommunikationsformen, die an Möglichkeiten, Geschwindigkeit und Grenzüberschreitung alles Bisherige in den Schatten stellen. Eine seltsame Veränderung unserer Sprache geht damit einher, die sich in Bereicherungen, aber auch Verstümmelungen äußert und mit der umzugehen wir ständig neu lernen müssen. Viele von uns begegnen dieser dynamischen, technologisch orientierten Entwicklung unserer Gesellschaft mit Skepsis oder gar Ablehnung, andere wiederum greifen sie euphorisch auf und treten ihr mit immer höheren Erwartungen, Herausforderungen und Risiken gegenüber.

Die Euphorie der zurückliegenden Jahre kumulierte bekanntlich in der New Economy, jener "Neuen Wirtschaft", die voll auf kommerzielle Nutzung der neuen Medien, auf gigantische Investitionen, auf hohe Wachstumsraten und geringes Inflationsrisiko setzte. Dass diese überzogene Rechnung nicht aufging, haben nicht nur reihenweise private wie öffentliche Unternehmensgründungen und Dotcom-Firmen erfahren, sondern auch Kapitalanleger und Aktionäre. Und heute? Glaubt man den täglichen Meldungen, so scheint die New Economy Schubkraft dadurch zu gewinnen, dass sie sich jetzt besonnen, realistisch, nach vernünftigen (alten?) kaufmännischen Regeln entwickelt. Dies betrifft vor allem neve Geschäftsmodelle und Informations- und Kommunikationsformen, die sich auf das Internet stützen, das sich offenbar als stabiler Faktor innerhalb aller ökonomischer Turbulenzen erwiesen hat. Dass sich die Kartographie als Informations- und Recherchemedium par excellence dieses welt- umspannenden Dienstes nicht entzieht, ist nur natürlich. Und zahlreiche Veröffentlichungen in den zurückliegenden $\mathrm{KN}$ Heften und einige im vorliegenden $\mathrm{Heft}$ zeugen davon.

So setzt sich zunächst F. Dickmann unter dem Titel "Mehr Schein als Sein?" kritisch mit der tatsächlichen Wahrnehmung kartengestützter Rauminformationen aus dem Internet auseinander. Ein Autorenteam um L. Hurni betrachtet Internet-basierte Atlasinformationssysteme (AIS) als logische Erweiterungen von Web-Client-Software zur Visualisierung räumlicher und zeitlicher Geodaten. Vorgestellt wird ein AIS am Beispiel GEOWARN zur Beobachtung und Uberwachung schlafender Vulkane.

A. Rappo, A. Cecconi und D. Burghardt zeigen, wie sich mit Hilfe der Fischaugenprojektion Karten auf kleinen Bildschirmen mobiler Geräte virtuell vergrößern lassen. Der Reproduktion großformatiger kartographischer Darstellungen, besonders aus GIS- oder Scandaten, und der Untersuchung entsprechender Farbkopiersysteme haben J. Schoppmeyer und H. Stumm ihren Beitrag gewidmet.

Mit der Frage, ob es heute noch Sinn macht, sich mit dem Thema so genannter Kartenverfälschungen in der DDR auseinander zu setzen, war die Schriftleitung der KN bereits beim Heft 6/2003 konfrontiert. Jetzt ist sie es wieder. Dass dieses Thema zu Kritik und Diskussion und damit zu einer besonderen Form der Kommunikation herausfordert, war zu erwarten. Und dass man ihm kompetent und sensibel begegnen kann, zeigen Anrufe, welche die Schriftleitung erreichten, ebenso wie der hier abgedruckte schriftliche Beitrag von A. Kowanda. Bedauerlich, vielleicht auch bezeichnend, ist, wenn offenbar "Verantwortliche" die Schriffleitung mit unangemessenen Vorwürfen und unsachlichen Außerungen konfrontieren, anstatt offen ihre Meinung zu Papier zu bringen. Denn dass die KN ein offenes Gesprächsforum sind, hat diese Zeitschrift mehrfach bewiesen.

Herzlich, Ihr 